\title{
Characterization of the normal subgroups of finite index for the group representation of a Cayley tree
}

\author{
F. H. Haydarov \\ National University of Uzbekistan, Tashkent, Uzbekistan \\ haydarov_imc@mail.ru
}

DOI 10.17586/2220-8054-2016-7-5-888-892

In this paper we give a characterization of normal subgroups for the group representation of the Cayley tree.

Keywords: Cayley tree, $G_{k}$-group, normal subgroup, homomorphism, epimorphism.

Received: 4 August 2016

Revised: 29 August 2016

\section{Introduction}

The method used for the description of Gibbs measures on Cayley trees is the method of Markov random field theory and recurrent equations of this theory, however, the modern theory of Gibbs measures on trees uses new tools such as group theory, information flows on trees, node-weighted random walks, contour methods on trees, nonlinear analysis. A very recently published book [1] discusses all above-mentioned methods for describing Gibbs measures on trees. In the configuration of physical system is located on a lattice (in our case on the graph of a group), then the configuration can be considered as a function defined on the lattice. There are many works devoted to several kind of partitions of groups (lattices) (see e.g. [1-5,7]).

One of the central problems in the theory of Gibbs measures is to study periodic Gibbs measures corresponding to a given Hamiltonian. For any normal subgroups $H$ of the group $G_{k}$, we define $H$-periodic Gibbs measures.

In Chapter 1 of [1] several normal subgroups were constructed for the group representation of the Cayley tree. In [6], we found full description of normal subgroups of index four and six for the group. In this paper, we continue this investigation and construct all normal subgroups of index eight and ten for the group representation of the Cayley tree.

Cayley tree. A Cayley tree (Bethe lattice) $\Gamma^{k}$ of order $k \geq 1$ is an infinite homogeneous tree, i.e., a graph without cycles, such that exactly $k+1$ edges originate from each vertex. Let $\Gamma^{k}=(V, L)$ where $V$ is the set of vertices and $L$ that of edges (arcs).

A group representation of the Cayley tree. Let $G_{k}$ be a free product of $k+1$ cyclic groups of the second order with generators $a_{1}, a_{2}, \ldots a_{k+1}$, respectively.

A one to one correspondence is known to exist between the set of vertices $V$ of the Cayley tree $\Gamma^{k}$ and the group $G_{k}($ see [1]).

To obtain this correspondence, we fix an arbitrary element $x_{0} \in V$ and let it correspond to the unit element $e$ of the group $G_{k}$. Using $a_{1}, \ldots, a_{k+1}$, we numerate the nearest-neighbors of element $e$, moving by positive direction. Next, we give the numeration for the nearest-neighbors of each $a_{i}, i=1, \ldots, k+1$ by $a_{i} a_{j}, j=1, \ldots, k+1$. Since all $a_{i}$ have the common neighbor $e$, we give to it $a_{i} a_{i}=a_{i}^{2}=e$. Other neighbors are numerated starting from $a_{i} a_{i}$ by the positive direction. We numerate the set of all the nearest-neighbors of each $a_{i} a_{j}$ by words $a_{i} a_{j} a_{q}, q=1, \ldots, k+1$, starting from $a_{i} a_{j} a_{j}=a_{i}$ by the positive direction. Iterating this argument, one gets a one-to-one correspondence between the set of vertices $V$ of the Cayley tree $\Gamma^{k}$ and the group $G_{k}$.

Any(minimally represented) element $x \in G_{k}$ has the following form: $\quad x=a_{i_{1}} a_{i_{2}} \ldots a_{i_{n}}$, where $1 \leq i_{m} \leq$ $k+1, m=1, \ldots, n$. The number $n$ is called the length of the word $x$ and is denoted by $l(x)$. The number of letters $a_{i}, i=1, \ldots, k+1$, that enter the non-contractible representation of the word $x$ is denoted by $w_{x}\left(a_{i}\right)$.

Proposition 1. [8] Let $\varphi$ be homomorphism of the group $G_{k}$ with the kernel $H$. Then $H$ is a normal subgroup of the group $G_{k}$ and $\varphi\left(G_{k}\right) \simeq G_{k} / H$, (where $G_{k} / H$ is a quotient group) i.e., the index $\left|G_{k}: H\right|$ coincides with the order $\left|\varphi\left(G_{k}\right)\right|$ of the group $\varphi\left(G_{k}\right)$.

Let $H$ be a normal subgroup of a group $G$. The natural homomorphism $g$ from $G$ onto the quotient group $G / H$ is given by the formula $g(a)=a H$ for all $a \in G$. Then, $\operatorname{Ker} \varphi=H$. 
Definition 1. Let $M_{1}, M_{2}, \ldots, M_{m}$ be some sets and $M_{i} \neq M_{j}$, for $i \neq j$. We call the intersection $\cap_{i=1}^{m} M_{i}$ contractible if there exists $i_{0}\left(1 \leq i_{0} \leq m\right)$ such that:

$$
\cap_{i=1}^{m} M_{i}=\left(\cap_{i=1}^{i_{0}-1} M_{i}\right) \cap\left(\cap_{i=i_{0}+1}^{m} M_{i}\right) .
$$

Let $N_{k}=\{1, \ldots, k+1\}$. The following Proposition describes several normal subgroups of $G_{k}$. Put

$$
H_{A}=\left\{x \in G_{k} \mid \sum_{i \in A} \omega_{x}\left(a_{i}\right) \text { is even }\right\}, A \subset N_{k} .
$$

Proposition 2. [1] For any $\emptyset \neq A \subseteq N_{k}$, the set $H_{A} \subset G_{k}$ satisfies the following properties: (a) $H_{A}$ is a normal subgroup and $\left|G_{k}: H_{A}\right|=2$;

(b) $H_{A} \neq H_{B}$, for all $A \neq B \subseteq N_{k}$;

(c) Let $A_{1}, A_{2}, \ldots, A_{m} \subseteq N_{k}$. If $\cap \cap_{i=1}^{m} H_{A_{i}}$ is non-contractible, then it is a normal subgroup of index $2^{m}$.

Theorem 1. [6]

1. The group $G_{k}$ does not have normal subgroups of odd index $(\neq 1)$.

2. The group $G_{k}$ has a normal subgroups of arbitrary even index.

\section{New normal subgroups of finite index}

\subsection{The case of index eight}

Definition 2. A group $G$ is called a dihedral group of degree 4 (i.e., $\left.D_{4}\right)$ if $G$ is generated by two elements a and $b$ satisfying the relations:

$$
o(a)=4, o(b)=2, \quad b a=a^{3} b
$$

Definition 3. A group $G$ is called a quaternion group (i.e., $\left.Q_{8}\right)$ if $G$ is generated by two elements a, b satisfying the relation:

$$
o(a)=4, a^{2}=b^{2}, b a=a^{3} b
$$

Remark 1. [8] $D_{4}$ is not isomorph to $Q_{8}$.

Definition 4. A commutative group $G$ is called a Klein 8-group (i.e., $\left.K_{8}\right)$ if $G$ is generated by three elements $a, b$ and $c$ satisfying the relations: $o(a)=o(b)=o(c)=2$.

Proposition 3. [8] There exist (up to isomorphism) only two noncommutative nonisomorphic groups of order 8

Proposition 4. Let $\varphi$ be a homomorphism of the group $G_{k}$ onto a group $G$ of order 8. Then, $\varphi\left(G_{k}\right)$ is isomorph to either $D_{4}$ or $K_{8}$.

Proof. Case 1 Let $\varphi\left(G_{k}\right)$ be isomorph to any noncommutative group of order 8. By Proposition $1, \varphi\left(G_{k}\right)$ is isomorph to either $D_{4}$ or $Q_{8}$. Let $\varphi\left(G_{k}\right) \simeq Q_{8}$ and $e_{q}$ be an identity element of the group $Q_{8}$. Then, $e_{q}=\varphi(e)=\varphi\left(a_{i}^{2}\right)=\left(\varphi\left(a_{i}\right)\right)^{2}$ where $a_{i} \in G_{k}, i \in N_{k}$. Hence, for the order of $\varphi\left(a_{i}\right)$, we have $o\left(\varphi\left(a_{i}\right)\right) \in\{1,2\}$. It is easy to check there are only two elements of the group $Q_{8}$ which order of element less than two. This is contradict.

Case 2 Let $\varphi\left(G_{k}, *\right)$ be isomorph to any commutative group $\left(G, *_{1}\right)$ of order 8 . Then, there exist distinct elements $a, b \in G$ such that $o(a)=o(b)=2$. Let $H=\{e, a, b, a b\}$. It's easy to check that $\left(H, *_{1}\right)$ is a normal subgroup of the group $\left(G, *_{1}\right)$. For $c \notin H$ we have $H \neq c H\left(c H=c *_{1} H\right)$. Hence $\varphi\left(G_{k}, *\right)$ is isomorph to only one commutative group $\left(c H \cup H, *_{1}\right)$. Clearly $\left(c H \cup H, *_{1}\right) \simeq K_{8}$.

The group $G$ has finit generators of the order two and $r$ is the minimal number of such generators of the group $G$ and without loss of generality, we can take these generators to be $b_{1}, b_{2}, \ldots b_{r}$. Let $e_{1}$ be an identity element of the group $G$. We define homomorphism from $G_{k}$ onto $G$. Let $\Xi_{n}=\left\{A_{1}, A_{2}, \ldots, A_{n}\right\}$ be a partition of $N_{k} \backslash A_{0}, \quad 0 \leq\left|A_{0}\right| \leq k+1-n$. Then, we consider homomorphism $u_{n}:\left\{a_{1}, a_{2}, \ldots, a_{k+1}\right\} \rightarrow\left\{e_{1}, b_{1} \ldots, b_{m}\right\}$ as

$$
u_{n}(x)=\left\{\begin{array}{l}
e_{1}, \text { if } x=a_{i}, i \in A_{0} \\
b_{j}, \text { if } x=a_{i}, i \in A_{j}, j=\overline{1, n} .
\end{array}\right.
$$


For $b \in G$, we denote that $R_{b}\left[b_{1}, b_{2}, \ldots, b_{m}\right]$ is a representation of the word $b$ by generators $b_{1}, b_{2}, \ldots, b_{r}, r \leq m$. We define the homomorphism $\gamma_{n}: G \rightarrow G$ by the formula

$$
\gamma_{n}(x)=\left\{\begin{array}{l}
e_{1}, \text { if } x=e_{1} \\
b_{i}, \text { if } x=b_{i}, i=\overline{1, r} \\
R_{b_{i}}\left[b_{1}, \ldots, b_{r}\right], \text { if } x=b_{i}, i \neq \overline{1, r}
\end{array}\right.
$$

We set:

$$
H_{\Xi_{n}}^{(p)}(G)=\left\{x \in G_{k} \mid l\left(\gamma_{n}\left(u_{n}(x)\right)\right) \text { is divisible by } 2 p\right\}, 2 \leq n \leq k-1 .
$$

Let $\left.\gamma_{n}\left(u_{n}(x)\right)\right)=\tilde{x}$. We introduce the following equivalence relation on the set $G_{k}: x \sim y$ if $\tilde{x}=\tilde{y}$. This relation is readily confirmed to be reflexive, symmetric and transitive.

Proposition 5. Let $\Xi_{n}=\left\{A_{1}, A_{2}, \ldots, A_{n}\right\}$ be a partition of $N_{k} \backslash A_{0}, \quad 0 \leq\left|A_{0}\right| \leq k+1-n$. Then $H_{\Xi_{p}}^{(p)}(G)$ is a normal subgroup of index $2 p$ of the group $G_{k}$.

Proof. For $x=a_{i_{1}} a_{i_{2}} \ldots a_{i_{n}} \in G_{k}$ it's sufficient to show that $x^{-1} H_{\Xi_{n}}^{(p)}(G) x \subseteq H_{\Xi_{n}}^{(p)}(G)$. Suppose that there exist $y \in G_{k}$ such that $l(\tilde{y}) \geq 2 p$. Let $\tilde{y}=b_{i_{1}} b_{i_{2}} \ldots b_{i_{q}}, q \geq 2 p$ and $S \stackrel{=}{=}\left\{b_{i_{1}}, b_{i_{1}} b_{i_{2}}, \ldots, b_{i_{1}} b_{i_{2}} \ldots b_{i_{q}}\right\}$. Since $S \subseteq G$ there exist $x_{1}, x_{2} \in S$ such that $x_{1}=x_{2}$. But this contradicts $\tilde{y}$, which is a non-contractible. Thus we have proved that $l(\tilde{y})<2 p$. Hence, for any $x \in H_{\Xi_{n}}^{(p)}(G)$ we have $\tilde{x}=e_{1}$. Next, we take any element $z$ from the set $x^{-1} H_{\Xi_{n}}^{(p)}(G) x$. Then, $z=x^{-1} h x$ for some $h \in H_{\Xi_{n}}^{(p)}(G)$. We have $\tilde{z}=\gamma_{n}\left(v_{n}(z)\right)=\gamma_{n}\left(v_{n}\left(x^{-1} h x\right)\right)=$ $=\gamma_{n}\left(v_{n}\left(x^{-1}\right) v_{n}(h) v_{n}(x)\right)=\gamma_{n}\left(v_{n}\left(x^{-1}\right)\right) \gamma_{n}\left(v_{n}(h)\right) \gamma_{n}\left(v_{n}(x)\right)$. From $\gamma_{n}\left(v_{n}(h)\right)=e_{1}$, we get $\tilde{z}=e_{1}$ i.e., $z \in H_{\Xi_{n}}^{(p)}(G)$. This completes the proof.

Since $A_{1}, A_{2}, A_{3} \subset N_{k}$ and $\cap_{i=1}^{3} H_{A_{i}}$ is non-contractible we denote the following set:

$$
\Re=\left\{\cap_{i=1}^{3} H_{A_{i}} \mid A_{1}, A_{2}, A_{3} \subset N_{k}\right\} .
$$

Theorem 2. For the group $G_{k}$, the following statement is hold:

$$
\begin{aligned}
& \left\{H \mid H \text { is a normal subgroup of } G_{k} \text { with }\left|G_{k}: H\right|=8\right\}= \\
& =\left\{H_{C_{0} C_{1} C_{2}}^{(4)}\left(D_{4}\right) \mid C_{1}, C_{2} \text { is a partition of } N_{k} \backslash C_{0}\right\} \cup \Re .
\end{aligned}
$$

Proof. Let $\phi$ be a homomorphism with $\left|G_{k}: \operatorname{Ker} \phi\right|=8$. Then by Proposition 2 we have $\phi\left(G_{k}\right) \simeq K_{8}$ and $\phi\left(G_{k}\right) \simeq D_{4}$.

Let $\phi: G_{k} \rightarrow K_{8}$ be an epimorphism. For any nonempty sets $A_{1}, A_{2}, A_{3} \subset N_{k}$, we give a one to one correspondence between operatornameKer $\left.\phi \mid \phi\left(G_{k}\right) \simeq K_{8}\right\}$ and $\Re$. Let $a_{i} \in G_{k}, i \in N_{k}$. We define following homomorphism corresponding to the set $A_{1}, A_{2}, A_{3}$ :

$$
\phi_{A_{1} A_{2} A_{3}}\left(a_{i}\right)=\left\{\begin{array}{l}
a, \text { if } i \in A_{1} \backslash\left(A_{2} \cup A_{3}\right) \\
b, \text { if } i \in A_{2} \backslash\left(A_{1} \cup A_{3}\right) \\
c, \text { if } i \in A_{3} \backslash\left(A_{1} \cup A_{2}\right) \\
a b, \text { if } i \in\left(A_{1} \cap A_{2}\right) \backslash\left(A_{1} \cap A_{2} \cap A_{3}\right) \\
a c, \text { if } i \in\left(A_{1} \cap A_{3}\right) \backslash\left(A_{1} \cap A_{2} \cap A_{3}\right) \\
b c, \text { if } i \in\left(A_{2} \cap A_{3}\right) \backslash\left(A_{1} \cap A_{2} \cap A_{3}\right) \\
a b c, \text { if } i \in A_{1} \cap A_{2} \cap A_{3} \\
e, \text { if } i \in N_{k} \backslash\left(A_{1} \cup A_{2} \cup A_{3}\right) .
\end{array}\right.
$$

If $i \in \emptyset$, then we'll accept that there is no index $i \in N_{k}$ for which that condition is not satisfied. It is easy to check Ker $\phi_{A_{1} A_{2} A_{3}}=H_{A_{1}} \cap H_{A_{2}} \cap H_{A_{3}}$. Hence $\left\{\operatorname{Ker} \phi \mid \phi\left(G_{k}\right) \simeq K_{8}\right\}=\Re$.

Now, we'll consider the case $\phi\left(G_{k}\right) \simeq D_{4}$. Let $\phi: G_{k} \rightarrow D_{4}$ be epimorphisms. Put

$$
C_{0}=\left\{i \mid \phi\left(a_{i}\right)=e\right\}, \quad C_{1}=\left\{i \mid \phi\left(a_{i}\right)=b\right\}, C_{2}=\left\{i \mid \phi\left(a_{i}\right)=a b\right\} .
$$


One can construct following homomorphism (corresponding to $C_{0}, C_{1}, C_{2}$ )

$$
\phi_{C_{0} C_{1} C_{2}}(x)=\left\{\begin{array}{l}
e, \text { if } \tilde{x}=e \\
a, \text { if } \tilde{x}=b_{2} b_{1} \\
a^{2}, \text { if } \tilde{x}=b_{2} b_{1} b_{2} b_{1} \\
a^{3}, \text { if } \tilde{x}=b_{2} b_{1} b_{2} b_{1} b_{2} b_{1} \\
b, \text { if } \tilde{x}=b_{1} \\
a b, \text { if } \tilde{x}=b_{2} \\
a^{2} b, \text { if } \tilde{x}=b_{2} b_{1} b_{2} \\
a^{3} b, \text { if } \tilde{x}=b_{2} b_{1} b_{2} b_{1} b_{2} .
\end{array}\right.
$$

Immediately, we conclude $\operatorname{Ker}\left(\phi_{C_{0} C_{1} C_{2}}\right)=H_{C_{0} C_{1} C_{2}}^{(4)}\left(D_{4}\right)$. We have constructed all homomorphisms $\phi$ on the group $G_{k}$ which $\left|G_{k}: \operatorname{Ker} \phi\right|=8$. Thus by Proposition 1, one gets:

$$
\left\{H|| G_{k}: H \mid=8\right\} \subseteq\left\{H_{C_{0} C_{1} C_{2}}^{(4)}\left(D_{4}\right) \mid C_{1}, C_{2} \text { is a partition of } N_{k} \backslash C_{0}\right\} \cup \Re .
$$

By Proposition 2 and Proposition 5, we can easily see that:

$$
\Re \cup\left\{H_{C_{0} C_{1} C_{2}}^{(4)}\left(D_{4}\right) \mid C_{1}, C_{2} \text { is a partition of } N_{k} \backslash C_{0}\right\} \subseteq\left\{H|| G_{k}: H \mid=8\right\} .
$$

The theorem is proved.

Corollary 1. The number of all normal subgroups of index 8 for the group $G_{k}$ is equal to: $8^{k+1}-6 \cdot 4^{k+1}+$ $3^{k+1}+9 \cdot 2^{k+1}-5$.

Proof. Number of elements of the set $H_{A} \subset G_{k}, \emptyset \neq A \subset N_{k}$ is $2^{k+1}-1$. Then $|\Re|=\left(2^{k+1}\right)\left(2^{k+1}-2\right)\left(2^{k+2}-3\right)$. Let $C_{0} \subset N_{k}$ be a fixed set and $\left|C_{0}\right|=p$. If $C_{1}, C_{2}$ is a partition of $N_{k} \backslash C_{0}$ then there are $2^{k-p+1}-2$ ways to choose the sets $C_{1}$ and $C_{2}$. Hence the cardinality of $\left\{H_{C_{0} C_{1} C_{2}}^{(4)}\left(D_{4}\right) \mid C_{1}, C_{2}\right.$ is a partition of $\left.N_{k} \backslash C_{0}\right\}$ is equal to

$$
\left(2^{k+1}-2\right) C_{k+1}^{0}+\left(2^{k}-2\right) C_{k+1}^{1}+\ldots+2 C_{k+1}^{k-1}=3^{k+1}-2^{k+2}+1 .
$$

Since $\Re$ and $\left\{H_{C_{0} C_{1} C_{2}}^{(4)}\left(D_{4}\right)\right\} \mid C_{1}, C_{2} \subset N_{k}$ are disjoint sets, the cardinality of the union of these sets is $8^{k+1}-$ $6 \cdot 4^{k+1}+3^{k+1}+9 \cdot 2^{k+1}-5$.

\subsection{Case of index ten}

Let the group $R_{10}$ be generated by the permutations:

$$
\pi_{1}=(1,2)(3,4)(5,6), \pi_{2}=(2,3)(4,5) .
$$

Proposition 6. Let $\varphi$ be a homomorphism of the group $G_{k}$ onto a group $G$ of order 10. Then, $\varphi\left(G_{k}\right)$ is isomorph to $R_{10}$.

Proof. Let $(G, *)$ be a group and $|G|=10$. Suppose there exist an epimorphism from $G_{k}$ onto $G$. It is easy to check that there are at least two elements $a, b \in G_{k}$ such that $o(a)=o(b)=2$. If $a * b=b * a$, then $(H, *)$ is a subgroup of the group $(G, *)$, where $H=\{e, a, b, a * b\}$. Then, by Lagrange's theorem, $|G|$ is divided by $|H|$ but 10 is not divided by 4 . Hence, $a * b \neq b * a$. We have $\{e, a, b, a * b, b * a\} \subset G$ If $G$ is generated by three elements, then there exist an element $c$ such that $c \notin\{e, a, b, a * b, b * a\}$. Then, the set $\{e, a, b, a * b, b * a, c, c * a, c * b, c * a * b, c * b * a\}$ must be equal to $G$. Since $G$ is a group, we get $a * b * a=b$ but from $o(a)=2$ the last equality is equivalent to $a * b=b * a$. This is a contradiction. Hence, by Lagrange's theorem it is easy to see:

$$
G=\{e, a, b, a * b, b * a, a * b * a, b * a * b, a * b * a * b, b * a * b * a, a * b * a * b * a\},
$$

where $o(a * b)=5$. Namely $G \simeq R_{10}$. This completes the proof.

Theorem 3. For the group $G_{k}$, the following statement is holds:

$\left\{H|| H\right.$ is a normal subgroup of $G_{k}$ with $\left.\left|G_{k}: H\right|=10\right\}=$

$$
=\left\{H_{B_{0} B_{1} B_{2}}^{(5)}\left(R_{10}\right) \mid B_{1}, B_{2} \text { is a partition of the set } N_{k} \backslash B_{0}\right\} \text {. }
$$


Proof. Let $\phi$ be a homomorphism with $\mid G_{k}$ : Ker $\phi \mid=10$. By Proposition $6 \phi\left(G_{k}\right) \simeq R_{10}$ and by Proposition 5 we can easily see:

$$
\left\{H_{B_{0} B_{1} B_{2}}^{(5)}\left(R_{10}\right) \mid B_{1}, B_{2} \text { is a partition of the set } N_{k} \backslash B_{0}\right\} \subset\left\{H|| G_{k}: H \mid=10\right\} .
$$

Let $\varphi: G_{k} \rightarrow R_{10}$ be epimorphisms. We denote:

$$
B_{0}=\left\{i \mid \varphi\left(a_{i}\right)=e\right\}, \quad B_{1}=\left\{i \mid \varphi\left(a_{i}\right)=a, B_{2}=\left\{i \mid \varphi\left(a_{i}\right)=b\right\} .\right.
$$

Then, we can show this homomorphism (corresponding to $B_{1}, B_{2}, B_{3}$ ), i.e.,

$$
\phi_{B_{0} B_{1} B_{2}}(x)=\left\{\begin{array}{l}
e, \text { if } \tilde{x}=e \\
a, \text { if } \tilde{x}=b_{1} \\
b, \text { if } \tilde{x}=b_{2} \\
a * b, \text { if } \tilde{x}=b_{1} b_{2} \\
b * a, \text { if } \tilde{x}=b_{2} b_{1} \\
a * b * a, \text { if } \tilde{x}=b_{1} b_{2} b_{1} \\
b * a * b, \text { if } \tilde{x}=b_{2} b_{1} b_{2} \\
a * b * a * b, \text { if } \tilde{x}=b_{1} b_{2} b_{1} b_{2} \\
b * a * b * a, \text { if } \tilde{x}=b_{2} b_{1} b_{2} b_{1} \\
a * b * a * b * a, \text { if } \tilde{x}=b_{1} b_{2} b_{1} b_{2} b_{1} .
\end{array}\right.
$$

We have constructed all homomorphisms $\phi$ on the group $G_{k}$ which $\left|G_{k}: \operatorname{Ker} \phi\right|=10$. Hence:

$\left\{\operatorname{Ker} \phi|| G_{k}: \operatorname{Ker} \phi \mid=10\right\} \subset\left\{H_{B_{0} B_{1} B_{2}}^{(5)}\left(R_{10}\right) \mid B_{1}, B_{2}\right.$ is a partition of the set $\left.N_{k} \backslash B_{0}\right\}$.

By Proposition 1:

$$
\left\{H|| G_{k}: H \mid=10\right\}=\left\{H_{B_{0} B_{1} B_{2}}^{(5)}\left(R_{10}\right) \mid B_{1}, B_{2} \text { is a partition of the set } N_{k} \backslash B_{0}\right\} .
$$

The theorem is proved.

Corollary 2. The number of all normal subgroups of index 10 for the group $G_{k}$ is equal to $3^{k+1}-2^{k+2}+1$.

Proof. The proof of this Corollary is similar to proof of Corollary 1.

\section{Acknowledgements}

I am deeply grateful to Professor U. A. Rozikov for the attention to my work.

\section{References}

[1] U.A. Rozikov. Gibbs measures on a Cayley trees, World Sci. Pub, Singapore, 2013.

[2] Cohen D.E., Lyndon R.C. Free bases for normal subgroups of free groups, Trans. Amer. Math. Soc., 1963, 108, P. 526-537.

[3] Ganikhodjaev N.N., Rozikov U.A. Description of periodic extreme Gibbs measures of some lattice model on the Cayley tree, Theor. Math. Phys., 1997, 111, P. 480-486.

[4] G.I. Botirov. Functional equations for the Potts model with competing interactions on a Cayley tree. Nanosystems: physics, chemistry, mathematics, 2016, 7(3), P. 401-404.

[5] Young J.W. On the partitions of a group and the resulting classification, Bull. Amer. Math. Soc., 1927, 33, P. 453-461.

[6] U.A. Rozikov, F.H. Haydarov. Normal subgroups of finite index for the group represantation of the Cayley tree, TWMS Jour. Pure. Appl. Math., 2014, 5, P. 234-240.

[7] Kurosh A.G. Group theory, Akademic Verlag, Berlin, 1953.

[8] D.S. Malik, J.N. Mordeson, M.K. Sen. Fundamentals of Abstract Algebra, McGraw-Hill Com., 1997. 